Published online 2017 April 13.

Abstract

\title{
Management of Loco-Regional Interventions in HCC
}

\author{
Hazhir Saberi ${ }^{1, *}$ \\ ${ }^{1}$ Radiology Professor of Tehran University of Medical Sciences \\ "Corresponding author: Hazhir Saberi, Radiology Professor of Tehran University of Medical Sciences. E-mail: hazhir_saberi@hotmail.com
}

Received 2016 December 21; Accepted 2017 February 08.

\begin{abstract}
Some patients are more susceptible to HCC (like HBV, Cirrhosis and ...). In these patientsscreening imaging (ultrasound) is highly recommended. In positive cases next step would be 4-phasic CT scan or dynamic MRI for confirm diagnosis and also staging. If there are typical signs of HCC (wash in, wash out), biopsy is not necessary in susceptible populations. Gold standard treatment of HCC is surgery (resection or liver transplantation). In thosecases which are not candidate for surgery, Loco-Regional treatments are key players. The mostnoticeable of these are: TACE (Trans Arterial Chemoembolization) and Local Ablations (RF, Microwave, Alcohol injections and ...). Site and Size of tumors should be considered in decision forablative techniques. Limitations for RF are 1) tumors more than $3 \mathrm{~cm}$ or 2) subcapsular locationor 3) neighboring to large vessels, but there are less limitations in Microwave. TACE is one of thetreatment of choice in cases which are not candidate for surgery or thermal ablations (intermediate stage). There are also some limitations for TACE which the most important one is decompensated cirrhosis (child B8, 9 and C). In this technique there are two factors (ischemia and chemotherapy) attenuating the tumors with synergistic effect. Generally TACE has a palliative situation in the guidelines but could be used as a bridge for curative plan (surgery or liver transplantation) alone or in combination with ablation techniques.
\end{abstract}

This is an abstract presented in the 33rd Iranian congress of radiology (ICR) and the 15th congress of Iranian radiographic science association (IRSA). 